\title{
Single potential analysis of cavernous electric activity - a possible diagnosis of autonomic impotence?
}

\author{
C. G. Stief*, M. Djamilian, F. Schaebsdau, M.C. Truss, R.W. Schlick, J. H. Abicht, E. P. Allhoff, and U. Jonas \\ Department of Urology, Hannover Medical School, Konstanty-Gutschow-Strasse 8, D-3000 Hannover 61, Federal Republic of Germany
}

\begin{abstract}
Summary. The aim of our study was to examine the cavernous smooth-muscle electric activity in normal and impotent patients as well as in those with (presumably) welldefined neurologic lesions. Single potential analysis of cavernous electric activity (SPACE) was done in 112 consecutive impotent patients, 34 normal patients, and 19 patients referred especially for SPACE. In the normal patients, similar potentials with a mean duration of $9.5 \mathrm{~s}$, a mean amplitude of $153 \mu \mathrm{V}$ and a mean polyphasity of 8.5 were recorded (cutoff frequencies, $2-2000 \mathrm{~Hz}$ ), with cutoff frequencies set at $0.5-500 \mathrm{~Hz}$, the mean duration was $12.8 \mathrm{~s}$, amplitude was $444 \mu \mathrm{V}$ and the mean polyphasity was 13.8 Upper spinal cord lesions showed potentials of long duration as well as whips and bursts. Patients with lower motor-neuron lesions showed either short potentials of high amplitude or potentials of small amplitude. In $49 \%$ of the impotent patients, abnormal SPACE was found. Our results suggest that SPACE is a reproducible and minimally invasive method for the diagnosis of autonomic neurogenic impotence.
\end{abstract}

\section{Introduction}

The diagnosis of vasculogenic erectile dysfunction has been substantially refined by the introduction of intracavernous injection of vasoactive drugs. Penile arterial inflow can be assessed rather precisely by pulsed Doppler sonography [6] and cavernous venous outflow, by pharmacocavernosometry and cavernosography [7]. Standardized intracavernous injection of vasoactive drugs [9] enables a good overall consideration of the penile vascular status and of cavernous smooth-muscle function.

To elucidate the status of cavernous autonomic innervation, the somatosensory penile innervation was examined due to the lack of tests for the autonomic nervous system. Measurement of the latency of the bulbocaver-

* To whom correspondence should be addressed nous reflex (BCR-L) was mostly done $[4,8]$, but recent publications cast some doubts on the relevance of this procedure for autonomic neuropathy [5]. Gorm Wagner was the first investigator to approach this major drawback in the diagnosis of erectile dysfunction when he suggested the registration of cavernous smooth-muscle activity [10]. Recordings were done during flaccidity and during visual sexual stimulation $[2,10,11]$.

In the following we describe the registration of cavernous electric activity using data processing different from that published by Wagner, thus enabling possibly a minimally invasive, reproducible and refined diagnosis of autonomic cavernous neuropathy.

\section{Patients and methods}

Since the introduction of single potential analysis of cavernous electric activity (SPACE) into our routine diagnostic workup, 112 consecutive patients have been evaluated multidisciplinarily in our impotence clinic. In all patients, the minimal duration of impotence was 1 year. Our standard workup consisted of a history and physical examination, sexual history and psychometry, laboratory blood tests including the determination of testosterone and prolactin values, Doppler analysis of the penile arteries [3], standardized pharmacotesting [9], neurophysiological examination including BCR-L, SSEP [8] and SPACE. When indicated [9], pharmacocavernosometry and cavernosography or phalloarteriography were done.

A total of 11 patients had insulin-dependent diabetes for 5 years, 8 had primary erectile dysfunction, 5 had chronic renal failure and 1 had multiple sclerosis. In addition, 3 patients unerwent cystoprostatectomy; 3 , radical prostatectomy; 3 , abdominoperineal extirpation of the rectum; and 4, a complete traumatic lesion of the spinal cord above Th 11. In all, 2 patients had posttraumatic impotence, 4 underwent spinal cord surgery for prolapse and 1 had a hemipelvectomy.

A total of 34 patients with normal erectile function underwent SPACE; for the first 23 patients, cutoff frequencies were $2-2000 \mathrm{~Hz}$ and for the rest they were $0.5-500 \mathrm{~Hz}$ as well as $2-500 \mathrm{~Hz}$. Most of the normal patients had been treated in our uroligical clinic; they were extensively informed about the procedure and possible risks and gave written consent. An additional 19 patients from other departments or centers were referred especially for SPACE evaluation. The procedure as well as the indications for its application were approved by the university ethical committee. 


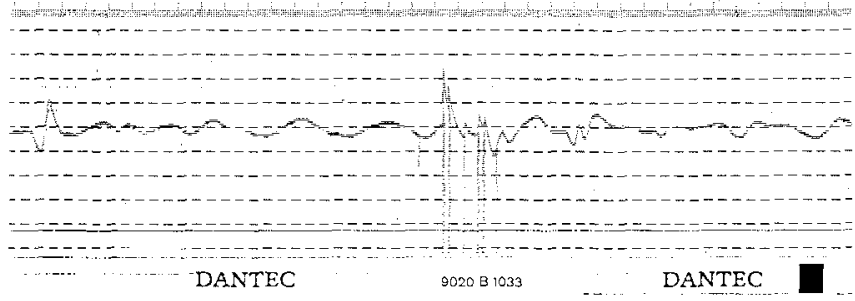

Fig. 1. This figure shows bursts in a 33-year-old patient with complete spinal cord injury at the level of Th $9 ; 1$ horizontal unit corresponds to $1 \mathrm{~s}$ and 1 vertical unit, to $50 \mu \mathrm{V}$

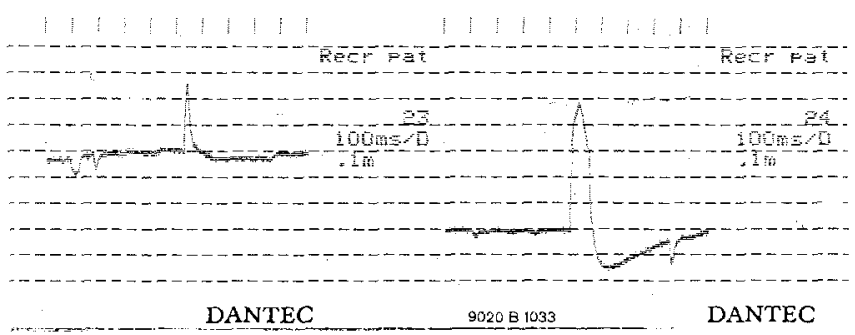

Fig. 2. Whips are the most frequent abnormal findings in patients with defined neurological lesions; 1 horizontal unit corresponds to $1 \mathrm{~s}$ and 1 vertical unit, to $100 \mu \mathrm{V}$
A coaxial needle electrode (Dantec $9013 \mathrm{~L}$ ) was inserted laterally into the cavernous body. The electrode was pushed forward into the proximal cavernous body until its tip was located in the center of the cavernous body. The potentials were processed by an electrophysiological unit (Dantec Neuromatic 2000; Spacecompact, Wiest, FRG). The signals were displayed continuously on a screen monitor and were simultaneously recorded (Dantec 21 F 02 Rec; after modifications with the intrinsic recorder of the Neuromatic); the paper speed was $5 \mathrm{~mm} / \mathrm{s}$. The cutoff frequencies were $2-2000 \mathrm{~Hz}$ for the first 71 patients and were then set at $0.5-500 \mathrm{~Hz}$. Amplification occurred mostly at $50 \mu \mathrm{V} / \mathrm{unit}$. For an exact description of the potentials, they were analyzed for (1) first depolarisation (plus or minus), (2) amplitude, (3) duration and (4) polyphasity (passages of the baseline).

Examination. Because all patients showed abnormal potential at up to $15 \mathrm{~min}$ after introduction of the needle electrode, SPACE evaluation was started $20 \mathrm{~min}$ after needle-electrode introduction. After a 25 -min registration of cavernous electric activity during flaccidity, audio-visual sexual stimulation (AVSS) was applied for another $25 \mathrm{~min}$, with the examiner leaving the room. At the end of AVSS, the examiner evaluated the erectile response by inspection, palpation and interrogation; the recording was then continued for another $10 \mathrm{~min}$.

Definitions. As in classic electromyography, negative depolarisations were defined as being in the up direction and positive depolarisations represented the down direction in reference to the baseline. Spikes are extremely short-lived depolarisations. Even when very brief intervals were displayed on the screen by the trigger technique, spikes remained lines and did not become waves. Bursts are groups of spikes (Fig. 1). Due to the instrument delay of the Dantec recorder, spikes and bursts could be documented only at a paper speed of $\geq 5 \mathrm{~cm} / \mathrm{s}$ in the first 71 patients. Whips are potentials with a very fast first phase of depolarisation and a slower repolarisation phase of concave shape (Fig. 2). Salves are potentials with a duration of $>20$ s (Fig. 3).

\section{Results}

The electric activity of the cavernous smooth muscles could not be registered in one impotent and two normal patients; in these three patients, only the pulse could be seen on the monitor. When we changed the lower cutoff frequency to $0.5 \mathrm{~Hz}$ in the second normal patient (overall patient 71), potentials were easily recorded. Starting with this patient, we therefore chose $0.5 \mathrm{~Hz}$ as the lower cutoff frequency. Lowering of the cutoff frequency from 2 to $0.5 \mathrm{~Hz}$ resulted in an increase in amplitude of about $80 \%-250 \%$ (Fig. 4); in some patients, some low-frequency depolarisations at the end of the potentials could be seen at $0.5 \mathrm{~Hz}$, but not at $2 \mathrm{~Hz}$. In these patients, potential length and polyphasity were consecutively changed as well.

In 31/33 normal patients with recorded activity, the potentials were similar; they started with either a positive depolarisation or a short-lived negative one followed by a positive depolarisation. At cutoff frequencies of $2-2000 \mathrm{~Hz}$, the duration of the potentials was $3-12$ (mean, 9.5-0.3) s, the amplitude was 60-250 (mean, $153 \pm 31) \mu \mathrm{V}$ and polyphasity was $5-11$ (mean, $8.5 \pm 0.3$ ). At cutoff frequencies of $0.5-500 \mathrm{~Hz}$, the duration was $8-18$ (mean, $12.8 \pm 2.8$ ) s, the amplitude was 250-750 (mean, $444 \pm 109) \mu \mathrm{V}$ and the polyphasity was $8-22$ (mean, 13.8 \pm 3.3 ). The shape of the potentials was very

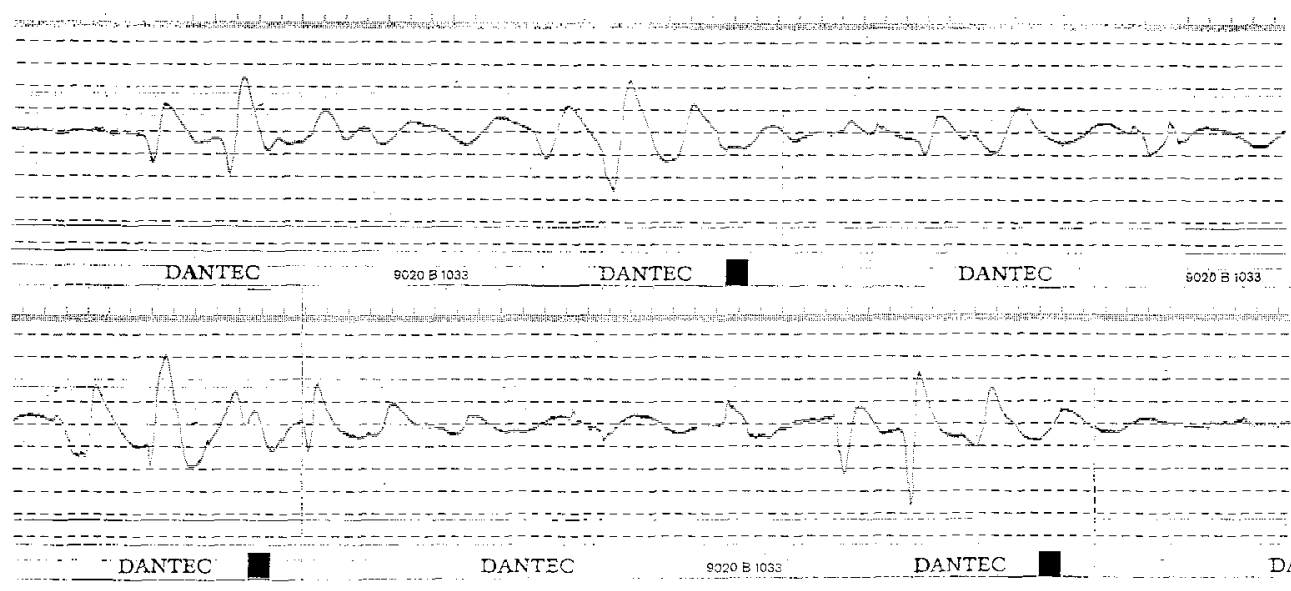

Fig. 3. Salves are pathophysiognomic for upper motor-neuron lesions, shown here in a 33-year-old patient (also see Fig. 1). The upper and lower lines were continuously recorded; 1 horizontal unit corresponds to $1 \mathrm{~s}$ and 1 vertical unit, to $50 \mu \mathrm{V}$ 


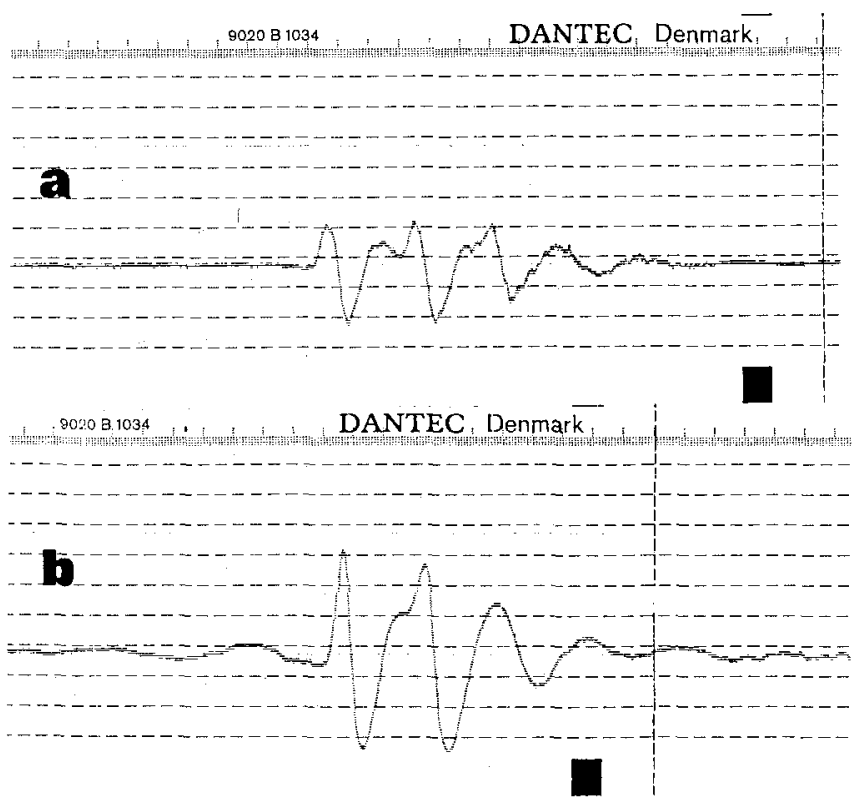

Fig. 4a,b. The effect of changing the cutoff frequencies from $2-2000 \mathrm{~Hz}$ (a) to $0.5-500 \mathrm{~Hz}$ (b); 1 horizontal unit corresponds to $1 \mathrm{~s}$ and 1 vertical unit, to $50 \mu \mathrm{V}$

uniform for the same individual (Fig. 5), although the frequency varied considerably, depending on the degree of relaxation of the patient. Spikes were seen at a frequency of about $0.4 / \mathrm{min}$ and both positive and negative spikes were recorded. The mean amplitude was $70 \mu \mathrm{V}$. We did not record bursts or whips in this group. To evaluate a possible influence of stress on the cavernous electric activity, SPACE was done about $1 \mathrm{~h}$ before surgery in two normal patients; in both cases, a wave-like electrical activity with no specific potential was recorded (Fig. 6).

All normal patients consented to AVSS. In all, 23/34 patients had a full erection during AVSS and 11/34 expe- rienced only tumescence. Comparison of the potentials during flaccidity and tumescence or erection revealed a uniform change: with increasing tumescence and rigidity, an increase in frequency of the potentials with a simultaneous decrease in amplitude and polyphasity was seen (Fig. 7). During full erection, potentials were recorded in all patients. In contrast to this, no potential (electrical silence) was recorded after intracavernous injection or papaverine and phentolamine of prostaglandin $E_{1}$.

In patients with lesions of the upper motor neuron (traumatic spinal cord injury above Th 11), salves lasting from $28 \mathrm{~s}$ to $>2$ min were recorded (Fig. 3). The amplitude of the potentials as well as the time between passages of the baseline were comparable with the potentials recorded in normal patients. Whips were numerous but bursts were less frequently recorded.

In patients with lesions of the lower motor neuron (e.g. after cystoprostatectomy), two different types of potential were recorded: on the one hand, short potentials with a significantly increased amplitude; on the other, short potentials with a significantly reduced amplitude (Fig. 8). Short whips, spikes and bursts were seen.

In $55 / 112$ patients $(49 \%)$ with erectile dysfunction, abnormal SPACE findings were recorded. Unstable first depolarisation, and unstable shape of the potentials and whips were recorded in all cases. Of these patients, 18 showed abnormal findings that alternated irregularly with normal potentials (Fig. 9). During AVSS, 9/21 patients with psychogenic impotence showed an increased frequency with otherwise unchanged potentials.

\section{Discussion}

Our findings show that single potentials of cavernous smooth-muscle activity can be recorded. The reproducible recordings and the similarity of the potentials in nor-
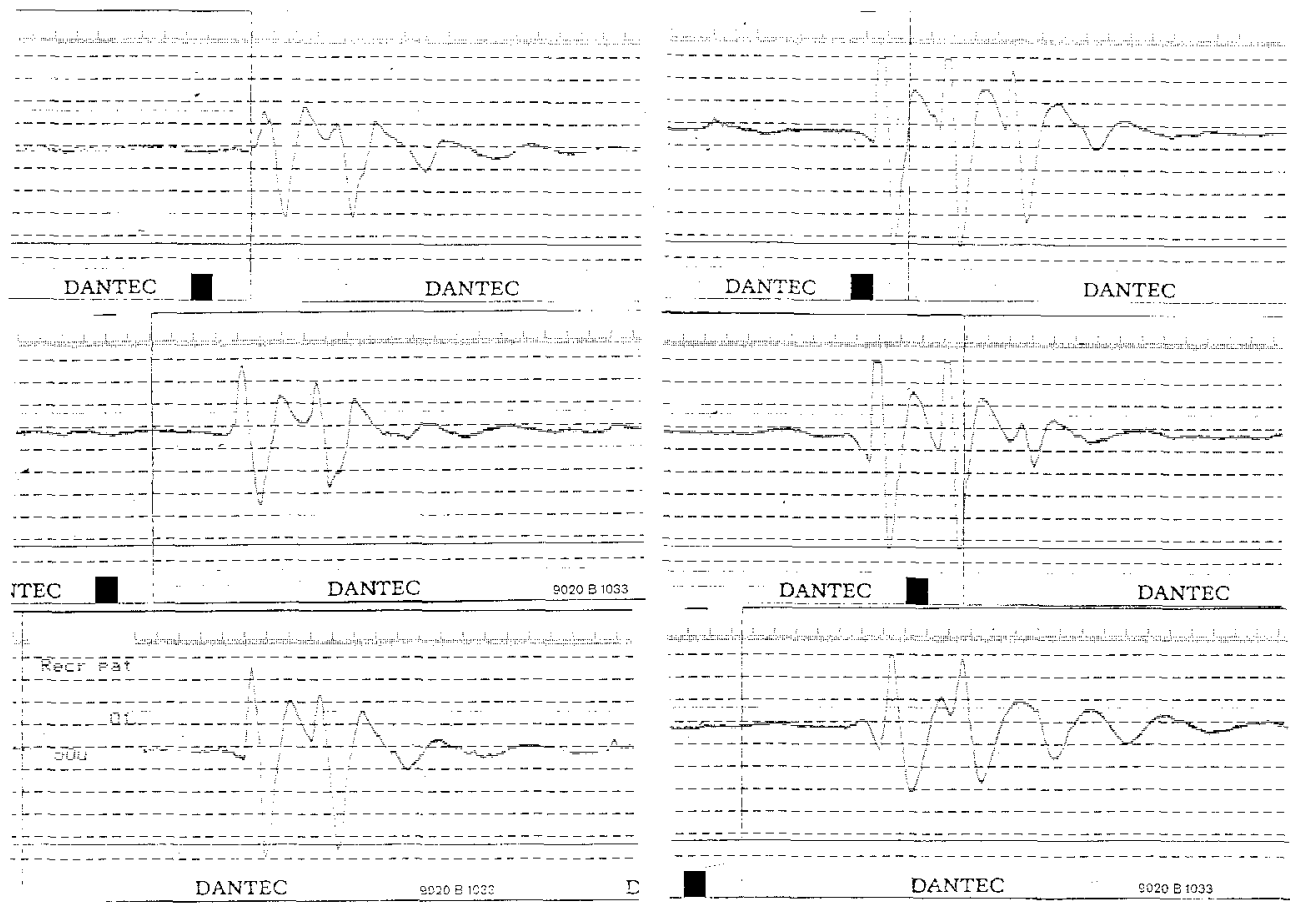

Fig. 5. Potentials recorded in the same patients were similar to each other. This figure shows 6 consecutive potentials in a 20 -year-old patient with congenital penile curvature; 1 horizontal unit corresponds to $1 \mathrm{~s}$ and 1 vertical unit, to $50 \mu \mathrm{V}$ 

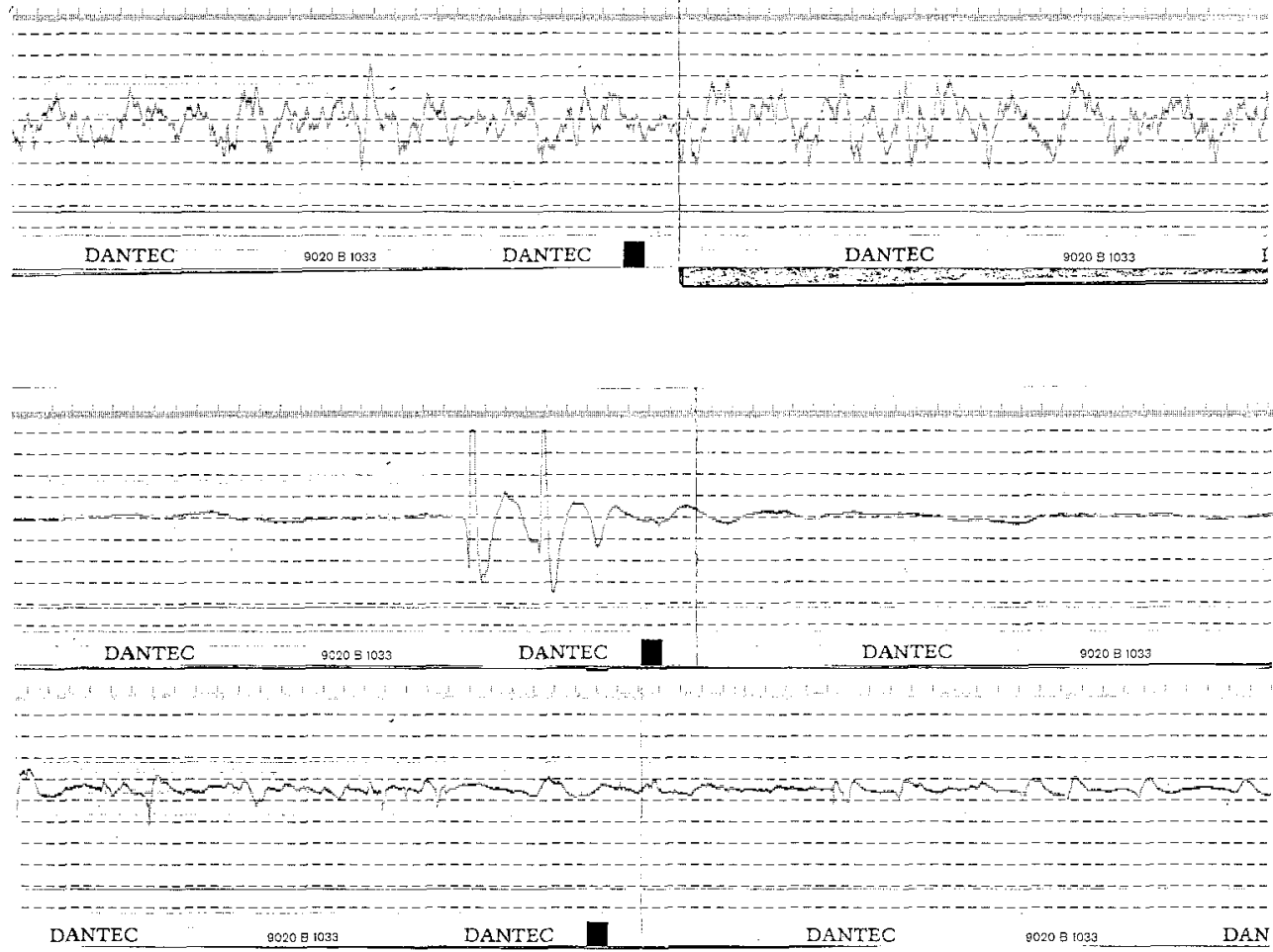

DANTEC 902081033 DANTEC
Fig. 6. Recording of a 29-year-old patient with varicocele, obtained $1 \mathrm{~h}$ prior to surgery; 1 horizontal unit corresponds to $1 \mathrm{~s}$ and 1 vertical unit, to $50 \mu \mathrm{V}$
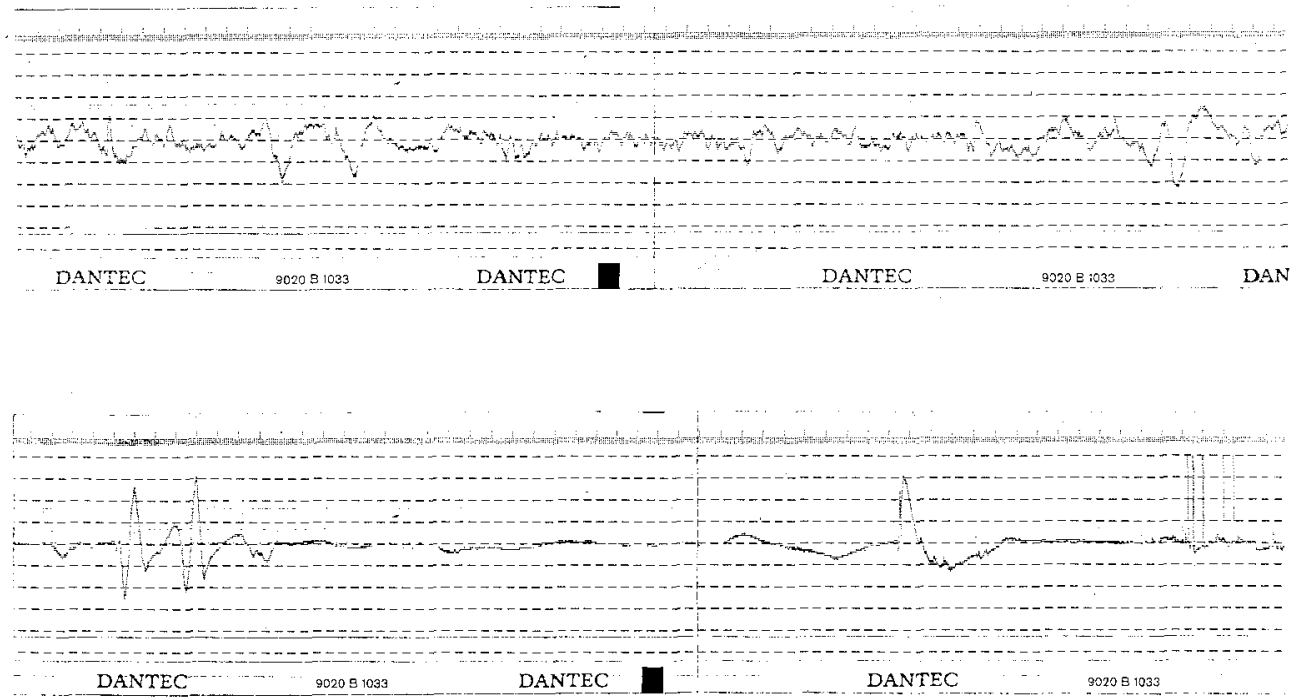

Fig. 7. The upper tracing shows a recording obtained during flaccidity in a 25-year-old normal patient (1 large potential) and the lower tracing shows the electrical activity (frequent, small potentials) recorded during AVSS; 1 horizontal unit corresponds to $1 \mathrm{~s}$ and 1 vertical unit, to $50 \mu \mathrm{V}$

Fig. 8. Recording of a 51-year-old patient who underwent abdominoperineal extirpation of the rectum 4 years ago; 1 horizontal unit corresponds to $1 \mathrm{~s}$ and 1 vertical unit, to $50 \mu \mathrm{V}$
Fig. 9. In a 33-year-old patient who had insulin-dependent diabetes for 18 years, normal potentials as well as whips and bursts were seen; 1 horizontal unit corresponds to $1 \mathrm{~s}$ and 1 vertical unit, to $50 \mu \mathrm{V}$ mal patients as well as the differences recorded during full erection with AVSS and with intracavernous injection of vasoactive drugs prove that the recordings are not artefacts. Different types of autonomic lesions may be differentiated by SPACE.

The patients in the groups with defined neurologic lesions were mostly younger and therefore presumably had a good cavernous smooth muscle. All but one of these patients achieved a full erection with the intracavernous injection of $\leq 0.5 \mathrm{ml}$ papaverine $(15 \mathrm{mg} / \mathrm{ml})$ and phentolamine $(0.5 \mathrm{mg} / \mathrm{ml})$; the mean dose was $0.31 \mathrm{ml}$. This full erectile response to a minimal dose of vasoactive drugs [9] strongly suggests an intact cavernous smooth muscle in these patients. Therefore, the alteration in the recording of cavernous electric activity was most probably due to the neurologic lesions of the patients and not to smooth-muscle degeneration. Nevertheless, further electron microscopic studies should elucidate the influence of smooth-muscle degeneration on SPACE.

Our findings in normal patients suggest that during flaccidity, the contractions of many cavernous smoothmuscle cells are synchronised by the sympathetic tone, which results in a potential of high amplitude and long duration. During sexual arousal and with increasing tumescence and rigidity, the sympathetic tone is dramatically reduced, producing non-synchronisation of the 
smooth-muscle cells. This results in potentials of decreased amplitude and duration, with an increase in frequency. In contrast to this physiological erection, that induced by vasoactive drugs is accompanied by electrical silence.

Our study showed a $49 \%$ rate of abnormal SPACE findings in 112 consecutive patients with erectile dysfunction. Even if we substract patients with a history of presumable neurogenic lesions (e.g. after cystoprostatectomy), we are left with a $34 \%$ rate of abnormal SPACE findings in impotent patients with a normal neurologic history. Compared with about $10 \%$ neurogenic factors in the etiology of impotence in the literature, this rate seems surprisingly high. Nevertheless, it must be taken into account that this rate was mostly based on the measurement of bulbocavernous reflex latency (BCR-L). In comparisons between the possibility of damage to the autonomic (possibly diagnosed by SPACE) vs the somatosensory system (diagnosed by BCR-L), it is well known that damages to the former may occur much earlier [1]. Furthermore, our (unpublished) electron microscopic studies showed pathologic findings in cavernous innervation in $40 \%$ of the patients evaluated.

We think that SPACE is a minimally invasive, reproducible diagnostic method for the evaluation of cavernous electric activity and, most probably, autonomic cavernous innervation. A diagnosis of cavernous smoothmuscle degeneration by SPACE seems possible.
This work was supported by a grant from the Deutsche Forschungsgemeinschaft (Sti 96/2-1).

\section{References}

1. Ellenberg M (1980) Development of urinary bladder dysfunction in diabetes mellitus. An Intern Med 92:321-324

2. Gerstenberg TC, Nordling J, Hald H, Wagner G (1989) Standardized evaluation of erectile dysfunction in 95 consecutive patients. $\mathbf{J}$ Urol 141:857-861

3. Jevtich MJ (1983) Non-invasive vascular and neurogenic tests in use for evaluation of angiogenic impotence. Int Angiogr 3:964-969

4. Laivas JG, Zayed AA, Labib KB (1981) The bulbocavernous reflex in urology. J Urol 126:197-200

5. Lavoisier P, Proulx J, Courtois F, Carful F (1989) Bulbocavernous reflex. J Urol 141:311-315

6. Lue TF, Hricak H, Marich KW, Tanagho EA (1985) Vasculogenic impotence evaluated by high-resolution ultrasonography and pulsed Doppler spectrum analysis. Radiology 155:777-781

7. Lue TF, Hricak H, Schmidt RA, Tanagho EA (1986) Functional evaluation of penile veins by cavernosography in papaverine-induced erection. J Urol 135:479-484

8. Siroky MB, Sax DS, Krane RJ (1979) Sacral signal tracing. J Urol 122:661-665

9. Stief CG, Bähren W, Gall H, Scherb W (1988) Functional evaluation of penile hemodynamics. J Urol 139:734-737

10. Wagner G, Gerstenberg T (1988) Human in vivo studies of electrical activity of corpus cavernosum. J Urol 139:327A

11. Wagner G, Gerstenberg T, Levin RJ (1989) Electrical activity of corpus cavernosum during flaccidity and erection of the human penis. J Urol 142:723-727 